\title{
DOMAIN PATTERN EVOLUTION IN POLARIZATION REVERSAL OF TELLURIC ACID AMMONIUM PHOSPHATE CRYSTALS
}

\author{
K. MATYjaseK ${ }^{a}$ AND Z. CZAPLA ${ }^{b}$ \\ ${ }^{a}$ Institute of Physics, Technical University of Szczecin \\ Al. Piastów 48/49, 70-311 Szczecin, Poland \\ ${ }^{b}$ Institute of Experimental Physics, University of Wrocław \\ Pl. M. Borna 9, 50-204 Wrocław, Poland \\ (Received March 14, 2000; in final form April 20, 2000)
}

The domain nucleation and growth during switching process in telluric acid ammonium phosphate crystals has been investigated by nematic liquid crystal decoration technique. The domain configuration arising during polarization reversal has been discussed, considering that the type of nucleation is the controlling factor in the propagation of the domain walls.

PACS numbers: 77.80.Dj

\section{Introduction}

Telluric acid ammonium phosphate (TAAP) having the chemical formula $\mathrm{Te}(\mathrm{OH})_{6} \cdot 2 \mathrm{NH}_{4} \mathrm{H}_{2} \mathrm{PO}_{4} \cdot\left(\mathrm{NH}_{4}\right)_{2} \mathrm{HPO}_{4}$ undergoes the phase transition at $321 \mathrm{~K}$ changing its symmetry from the centrosymmetric class $2 / m$ to the polar class $m[1]$.

Currently most of what is known about the TAAP crystals relates to dielectric properties, little is known about their domain kinetics. The static domain structure has been observed by the etching technique [2]. This method simultaneously reveals the dislocations and other lattice imperfections. The first switching current measurement of ferroelectric polarization reversal in TAAP has been reported in Ref. [3].

Here we communicate the peculiarities of the domain structure dynamics in the TAAP crystals at room temperature.

\section{Experimental}

The domain structure has been observed using the nematic liquid crystal (NLC) method described in Refs. [4, 5]. To observe the domains we used the nematic phase of $p$-methoxybenzylidene- $p$ - $n$-butylaniline (MBBA) and $p$-ethoxybenzylidene- $p$ - $n$-butylaniline (EBBA) mixtures. After supplying a sample (coated with NLC-layers on its surfaces) with transparent electrodes the electric field was applied and the observation of domain pattern was carried out using a polarization 
microscope. The domain pattern was registered with a digital video camera and a computer processed the image. The observation of the domains was carried out on (101) natural face of the crystal, which is approximately normal to the polarization direction [1].

\section{Results}

The static domain structure can be delineated with a high resolution by rubbing the crystal surface on warm, wet filter paper. Figure 1 shows the etched patterns of the domain structure observed on the (101) crystal face. Our observations show that the TAAP crystals grown from an aqueous solution at room temperature are highly inhomogeneous. An as-grown crystal sample is often a single domain in which small domains of opposite polarity are randomly distributed. In some part of the crystal the plate-like domains elongated in the $b$-direction are seen (Fig. 1a). After thermal annealing at a temperature of $20 \mathrm{~K}$ above the Curie point for a few hours and then slowly cooling to room temperature, the domain structure appears

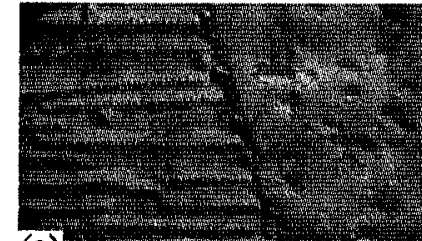

(a)

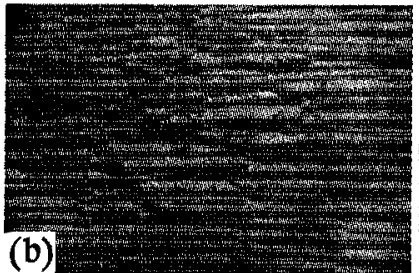

(b)

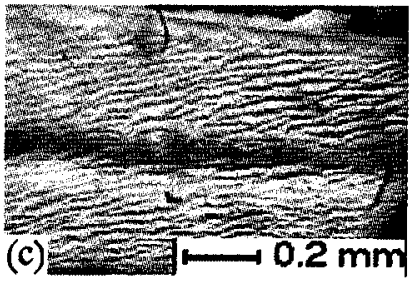

Fig. 1. Domain patterns in TAAP at room temperature revealed by etching with water; (a) as-grown sample; $(\mathrm{b}, \mathrm{c})$ annealed above $T_{\mathrm{c}}$.

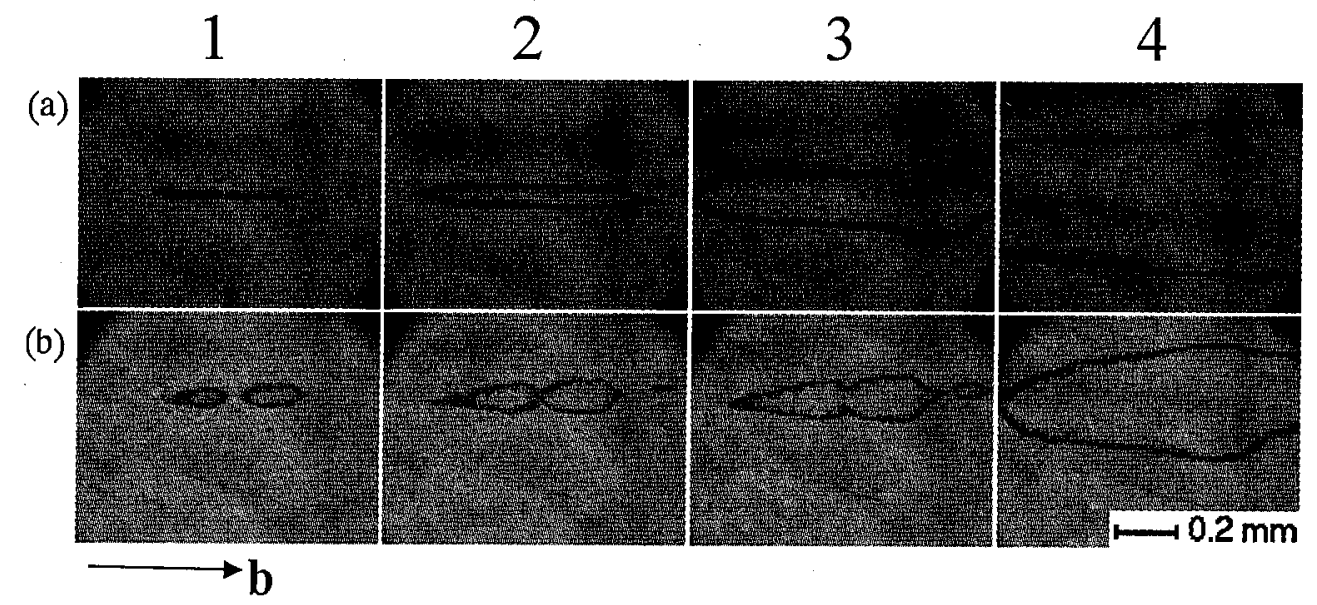

Fig. 2. Domain pattern evolution under two different electric fields applied at a time $t=0$ to the previously monodomenized crystal sample with the initial plate-like domain structure (as shown in Fig. 1b). The time from the moment of applying $E:$ (a) $80 \mathrm{kV} / \mathrm{m}$, $1-2 \mathrm{~s}, 2-9 \mathrm{~s}, 3-25 \mathrm{~s}, 4-60 \mathrm{~s} ;$ (b) $105 \mathrm{kV} / \mathrm{m}, 1-2 \mathrm{~s}, 2-5 \mathrm{~s}, 3-7 \mathrm{~s}, 4-11 \mathrm{~s}$. 
in various forms in different growth pyramids (see Fig. 1b, c). Our investigation shows that an initial domain structure in the crystal sample determines the shape of growing domains only in a weak external field. Figure 2 shows the time evolution of the domain structure under two different electric field strengths applied to the crystal sample $(0.5 \mathrm{~mm}$ thick) with the initial plate-like domain structure (as shown in Fig. 1b). One can see that the electric field of the order of threshold nucleation field creates the plate-like domains elongated in the $b$-direction (see Fig. 2a). In this range of $E$ the sideways wall motion is quite non-uniform because the walls are impeded by defects, which can cause a definite stabilization of domain walls at given sites of the sample. When the electric field increases, new circular domains are nucleated, but anisotropy of the domain wall motion results in the elliptical domains (Fig. 2b). Nearly the same domain pattern evolution (as shown in

(a)
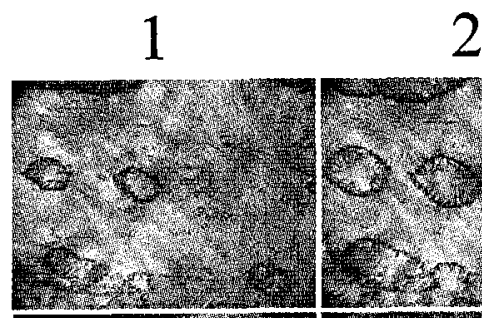

2

3

4

(b)

(c)
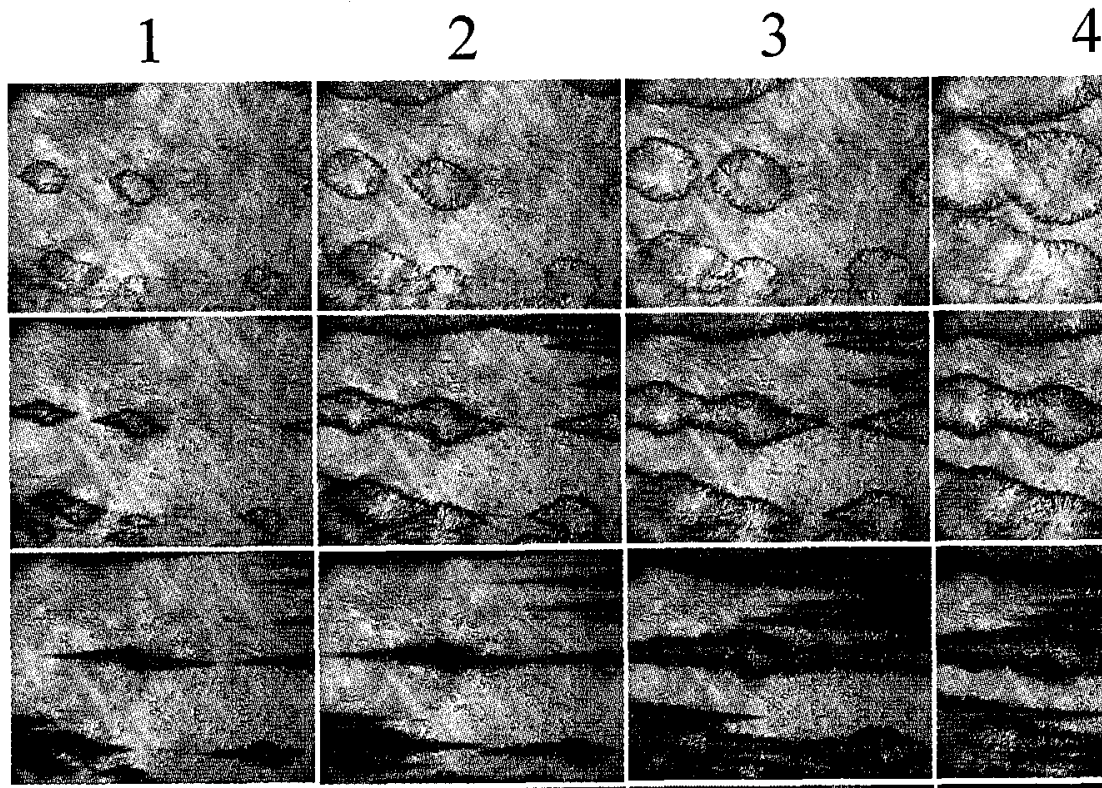

(d)
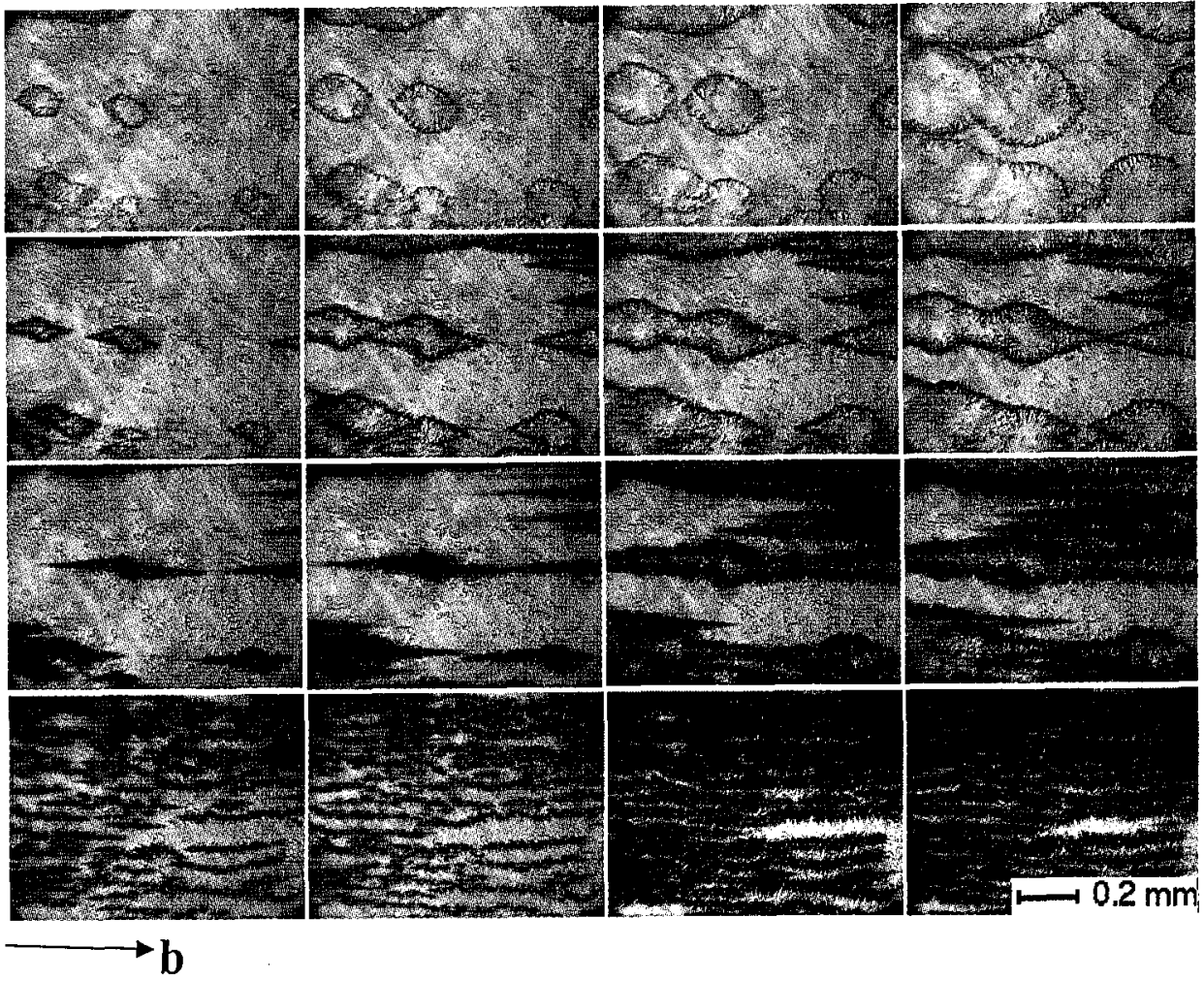

Fig. 3. Domain pattern evolution under four different electric field strengths applied at $t=0$ to the previously monodomenized crystal sample with the initial domain structure as shown in Fig. 1c. The time from the moment of applying $E:(a) 125 \mathrm{kV} / \mathrm{m}, 1-0.4 \mathrm{~s}$, $2-0.6 \mathrm{~s}, 3-1 \mathrm{~s}, 4-1.8 \mathrm{~s} ;$ (b) $135 \mathrm{kV} / \mathrm{m}, 1-0.12 \mathrm{~s}, 2-0.36 \mathrm{~s}, 3-0.50 \mathrm{~s}$, $4-0.70 \mathrm{~s}$; (c) $145 \mathrm{kV} / \mathrm{m}, 1-0.08 \mathrm{~s}, 2-0.12 \mathrm{~s}, 3-0.20 \mathrm{~s}, 4-0.26 \mathrm{~s} ;$ (d) $160 \mathrm{kV} / \mathrm{m}$, $1-0.02 \mathrm{~s}, 2-0.04 \mathrm{~s}, 3-0.06 \mathrm{~s}, 4-0.10 \mathrm{~s}$. 
Fig. 2b) has been observed in a part of the crystal with the initial domain structure presented in Fig. 1c, when the electric field of the order of threshold nucleation field has been applied to the crystal. Even then, the circular domains are nucleated but because of anisotropy of the domain wall motion, individual domains become elongated in the $b$-direction. In a higher electric field the nucleation and growth of circular domains have been observed on the whole crystal surface disregarding the initial domain structure. Figure $3 \mathrm{a}$ shows the domain pattern evolution of circular domains observed in a part of the crystal with the initial domain structure presented in Fig. 1c. In the range of weak electric fields, in which circular domains are nucleated and grow, the nuclei come out at almost the same position of the crystal during the repeating switching and only at the beginning of the switching.

One would expect that a further increase in the electric field must lead only to the increase in concentration of new domain nuclei. However, this is only observed in a freshly annealed crystal after quenching the crystal from the paraelectric phase. During the ageing process the number of nucleated domains decreases with the passing of time. Moreover, in the aged crystal (several hours after annealing) the edge of existing domains is more favourable for the nucleation and growth of new domains than elsewhere. We found the boundaries to be often of a zigzag type, as a result of the preferential formation of new nuclei, which appear adjacent to the moving walls (see Fig. 3b). For a sufficiently strong field, the individual domains transform into a band, as a result of the preferential formation of new nuclei along the $b$-direction (Fig. 3c). In stronger fields, the intensive domain nucleation occurs on the whole crystal plate. As can be seen in Fig. 3d the domain nuclei, which are distributed in a random manner on the crystal surface in weak dc-fields, are aligned into rows (mostly parallel to the $b$-direction) in strong fields. The number of domain nuclei in rows in the $b$-direction is so large that they coalesce in this direction in an early stage of the switching process and thus stripes are formed and expand. While increasing the electric field, the density of domain nuclei in each row and the number of rows increase so that they become optically not distinguished.

In order to explain such peculiarities of the domain structure dynamics, we have measured the velocity of the sideways domain wall motion as a function of electric field strength. To have independently growing domains (domains spatially well separated from other nuclei) the experiments were carried out two days after the annealing of the sample. At that time the domain structure changed reproducibly by repeated experiments.

It should be noted that the influence of the ageing process on the domain structure dynamics has been examined in doped triglycine sulphate [6] and $\left(\mathrm{CH}_{3} \mathrm{NH}_{3}\right)_{5} \mathrm{Bi}_{2} \mathrm{Br}_{11}$ [7] crystals.

As the velocity of the wall is anisotropic and inhomogeneous, the average velocity was measured at the same wall shift from the initial position in the $b$-direction. To obtain the relevant time interval required for the wall to move through a known distance, a stop-watch was used in the range of weak fields and a digital video camera in higher fields, respectively.

The data obtained for the crystal with the initial plate-like domain structure are illustrated in Fig. 4. For weak electric fields (plate-like domains) the field dependence of the average wall velocity $V$ is approximately linear. In higher electric 


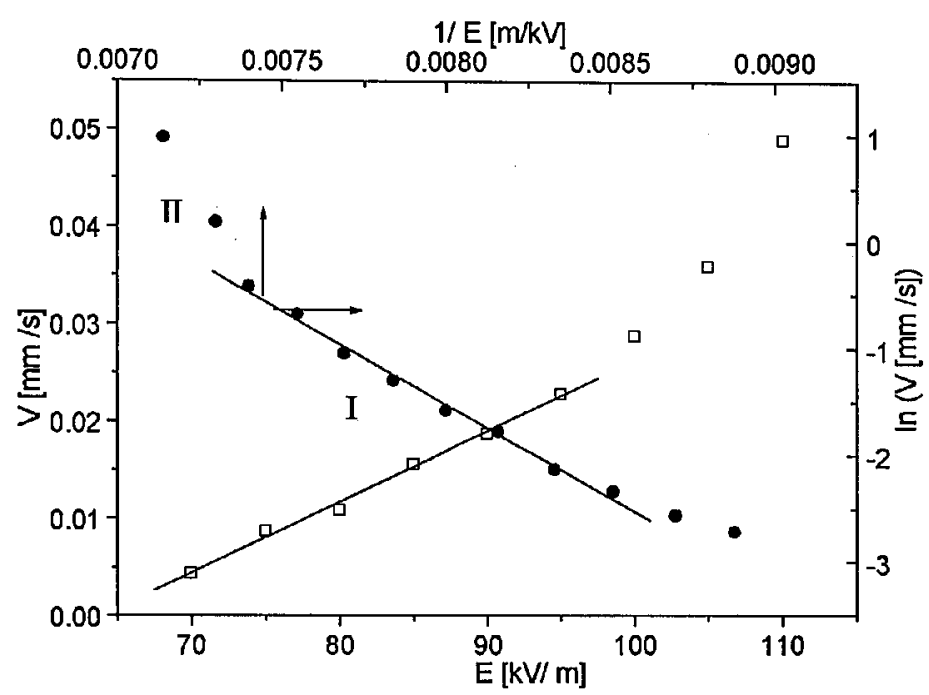

Fig. 4. An average sideways wall velocity of $180^{\circ}$ domain walls in TAAP as a function of electric field strength (as shown in open squares). Logarithmic plot of $V$ vs. $1 / E$ is shown by $\bullet$.

fields (circular domains) the domain wall velocity is approximated by the exponential field dependence described by the following relation $V=V_{0} \exp (-\delta / E)$ with the activation field of domain wall motion $\delta_{\mathrm{I}}=1736 \mathrm{kV} / \mathrm{m}$. The $\ln V$ versus $1 / E$ plots show two linear parts which correspond to different activation fields related to various shapes of domain walls (circular - in the region I and zigzag-type - in the region II). In the region II the wall displacement rate is found to increase substantially, as a result of the formation of new nuclei adjacent to the moving domain wall. However, the data are not reproducible because the process of nucleation is not the same in every cycle.

Measurements made on a different crystal, or on the same being at different stages of the ageing process, can produce the values of $\delta$ different from that given above as much as $50 \%$.

\section{Discussion of results}

The domain configuration arising during polarization reversal can be explained assuming that the mechanism of domain growth is similar to the mechanism of crystal growth. The theory of crystal growth $[8,9]$ assumes that at weak oversaturation the growth of the crystal is due to the one-dimensional nucleation steps at the phase boundary. The motion of these steps along the boundary results in layer after layer growth of the crystal. In this case the linear dependence of growth velocity is observed and crystals with regular faces are obtained. At large oversaturation the crystal is growing through the two-dimensional nucleation at the phase boundary. Due to the disorder nature of this process the velocity of growth is proportional to the rate of nucleation, thus the exponential dependence 
of the growth velocity is observed and no preference in crystal orientation is to be seen.

By analogy with the theory of crystal growth it is assumed that the switching process is determined by the oversaturation degree, which corresponds in ferroelectrics to the magnitude of the electric field acting on the domain boundary. This model satisfactorily explains the domain pattern evolution in lead germanate [10] and alkylammonium halogenobismuthate [11] crystals.

It is supposed that the mechanism of the domain growth in weak fields is suitable for the one-dimensional nucleation steps at a domain wall. The main problem in the one-dimensional mechanism is the problem of step origin. Nakamura [12] pointed out that the mechanism of the domain nucleation in weak fields is closely related to the crystal imperfections of dislocation lines. A small cylindrical domain is anchored to each dislocation line, and could act as a nucleus of a reversed domain. Our observation confirmed that the domain switching starts at certain definite sites of the crystal, when a weak electric field is applied. This means that the latent nuclei are brought about from some defects existing in the crystal sample. The anisotropy of the surface energy must lead to the preferable motion of steps along one direction thus, layer after layer growth of domains of preferable orientation, parallel to the $b$-axis, like in the as-grown TAAP crystal takes place. With increasing the electric field the probability of nucleation of two-dimensional nuclei is higher and the individual domains expand two-dimensionally after the formation of cylindrical nuclei. Their orientation is determined by the anisotropy of the surface energy.

It has been shown that the electric field dependence of the probability for the nucleation of reversed domains can be of the form $\exp (-$ const $/ E)$, if $E$ is not too great (typically lower than twice coercive field measured from the $D-E$ hysteresis loop) $[13,14]$. This field dependence is of the same form as that found for the sideways wall motion, where $V=V_{0} \exp (-\delta / E)$ and therefore suggests that the nucleation rate rather than the growth rate is the controlling factor in the propagation of a domain wall. Thus the shape of domain depends only on the anisotropy of the activation field $\delta$ for the domain wall motion and that $\delta$ is proportional to the domain wall energy according to Miller-Weinreich model [15]. The observation of the zigzag shape of domain walls, arising from the preferential nucleation at the existing domain walls, is the evidence that the domain walls move by a multiple nucleation process of the form postulated in the model.

\section{Conclusions}

We have observed the real-time dynamics of the domain structure in TAAP during the switching process. In the low field regime $(E<140 \mathrm{kV} / \mathrm{m})$ the switching time is dominated by the sideways growth of initially nucleated domains; plate-like in weak fields and elliptical in higher fields. It was found that the field dependence of sideways wall velocity of plate-like domains is linear whereas of elliptical domains is exponential. In the high field regime $(E>160 \mathrm{kV} / \mathrm{m})$ the density of nucleated domains is so high that it nearly prevents the sideways movement of domain walls. In consequence, most of the polarization will be reversed during the forward growth and little during the subsequent sideways growth. 


\section{References}

[1] S. Guillot Gauthier, J.C. Peuzin, M. Olivier, G. Rolland, Ferroelectrics 52, 293 (1984).

[2] Y.F. Nicolau, Ferroelectrics 52, 281 (1984).

[3] K.S. Sangunni, M.N. Shashikala, H.L. Bhat, P.S. Narayanan, Ferroelectrics 94, 439 (1989).

[4] Y. Furuhata, K. Toriyama, Appl. Phys. Lett. 23, 361 (1973).

[5] N.A. Tikhomirova, L.N. Dontsova, S.A. Pikin, A.V. Ginsberg, P.V. Adomenas, Kristallografiya 23, 1239 (1978).

[6] A. Czarnecka, J. Stankowska, S. Mielcarek, Acta Phys. Pol. A 85, 849 (1994).

[7] K. Matyjasek, Ferroelectrics 238, 91 (2000).

[8] W.K. Burton, N. Cabrera, F.C. Frank, Philos. Trans. R. Soc. 243, 299 (1951).

[9] A.A. Chernov, Mod. Crystallogr. 3, 7 (1980).

[10] V.Ya. Shur, A.L. Gruverman, E.L. Rumyantsev, Ferroelectrics 111, 123 (1990).

[11] K. Matyjasek, R. Jakubas, Acta Phys. Pol. A 85, 835 (1994).

[12] T. Nakamura, J. Phys. Soc. Jpn. 15, 853 (1960).

[13] A.G. Chynoweth, Phys. Rev. 110, 1316 (1958).

[14] V. Gopalan, T.E. Mitchell, J. Appl. Phys. 83, 941 (1998).

[15] R.C. Miller, G. Weinreich, Phys. Rev. 117, 1460 (1963). 